\title{
Bifurcation of the tail current sheet and ion temperature anisotropy
}

\author{
P. L. Israelevich and A. I. Ershkovich \\ Department of Geophysics and Planetary Sciences, The Raymond and Beverly Sackler Faculty of Exact Sciences, Tel Aviv \\ University, Ramat Aviv, Israel
}

Received: 17 February 2008 - Accepted: 5 May 2008 - Published: 25 June 2008

\begin{abstract}
We investigated the relation between the geotail current sheet structure and the anisotropy of the ion temperature in the plasma sheet. Current density distribution in the geomagnetic tail is shown not to depend on the ratio between the parallel and perpendicular ion temperature. The tail current sheet bifurcation is controlled by non-gyrotropicity of plasma pressure: double peaked current density distribution is observed when the ion perpendicular temperature exhibits anisotropy, and the electric current density is stronger for larger ratio $\cdot\left(T_{\perp \text { max }}-T_{\perp \text { min }}\right) / T_{\perp}$. The current sheet thinning is accompanied by the perpendicular temperature anisotropy, and, generally, double-peaked current sheets are thinner than single-peaked sheets.
\end{abstract}

Keywords. Magnetospheric physics (Current systems; Magnetotail; Plasma sheet)

\section{Introduction}

The average configuration of the magnetic tail (e.g. Behannon, 1970; Fairfield, 1979) contains two lobes of magnetic field lines with opposite polarity, separated by the plasma sheet with dawn-to-dusk electric current. For decades, the study of the processes in the geomagnetic tail was based on the Harris model of the current sheet. Harris (1962) has obtained (in simple analytical form) one of possible selfconsistent solutions of Vlasov's equation describing the onedimensional layer of plasma confined between two regions of oppositely directed magnetic field. This model ignored such an essential fact as the presence of the magnetic field component normal to the current sheet which makes the problem three-dimensional. Also, it was never confirmed experimentally that the current carriers in the magnetospheric frame of reference are ions as it follows from the model.

Correspondence to: P. L. Israelevich

(peteri@post.tau.ac.il)
On the contrary, the geotail electric current is transferred by electrons (Israelevich et al., 2008). Even first experimental evidences that geotail current sheet often exhibits two offcentered maxima of electric current density (Sergeev et al., 1993; Hoshino et al., 1996) did not attract attention they deserve as they contradicted the common views on the current sheet structure. Only direct measurements of electric current density by Cluster system of satellites (e.g. Runov et al., 2003, 2004, 2005, 2006; Sergeev et al., 2003), which revealed a number of double peaked current sheet crossings, initiated reconsideration of the old current sheet model and stimulated theoretical and model studies of the current sheet structure as well as possible causes of its bifurcation (e.g. Sitnov et al., 2003; Zelenyi et al., 2003; Ricci et al., 2004; Genot et al., 2005; Camporeale and Lapenta, 2005).

First statistical analysis of bifurcated current sheets (Asano et al., 2005) has shown that they are rather common feature of the Earth's magnetosphere. Moreover, evidences for current sheet bifurcation were found for the Jovian magnetosphere (Israelevich and Ershkovich, 2006; Israelevich et al., 2007).

The existence of bifurcated current sheets has been predicted by Cowley (1978). According to his fluid model, rather modest values of plasma pressure anisotropy result in drastic change of the current sheet structure. For the case of $P_{\|}>P_{\perp}$, a thin, non-adiabatic current layer is formed in the center of the plasma sheet being embedded within broad adiabatic current distribution. When $P_{\| \mid}<P_{\perp}$, a minimum of the electric current density arises near the center of the sheet, terminated at its outer boundary by a spike in the current density. Recent Cluster observations of bifurcated current sheets put a new challenge for further theoretical studies of the geomagnetic tail current sheet. A number of new models have been proposed including ion dynamics (Zelenyi et al., 2003), ion-ion kink instability (Karimabaldi et al., 2003a, b), effects of plasma non-gyrotropicity (Sitnov et al., 2003), lower-hybrid drift instability (Ricci et al., 2004; Daughton

Published by Copernicus Publications on behalf of the European Geosciences Union. 
Table 1. Summary of observations.

\begin{tabular}{ccccc}
\hline Day & $\begin{array}{c}\text { Start time } \\
(\text { hh:mm:ss UT })\end{array}$ & $\begin{array}{c}\text { Start } X_{\mathrm{GSM}} \\
\left(R_{E}\right)\end{array}$ & $\begin{array}{c}\text { End time } \\
(\text { hh:mm:ss UT })\end{array}$ & $\begin{array}{c}\text { End } X_{\mathrm{GSM}} \\
\left(R_{E}\right)\end{array}$ \\
\hline 3 Aug 2001 & $08: 22: 28$ & -16.6 & $11: 59: 57$ & -16.4 \\
7 Aug 2001 & $23: 30: 16$ & -17.1 & $23: 59: 37$ & -17.1 \\
10 Aug 2001 & $12: 00: 14$ & -17.6 & $13: 59: 45$ & -17.5 \\
12 Aug 2001 & $15: 00: 10$ & -17.1 & $18: 59: 51$ & -18.0 \\
15 Aug 2001 & $07: 30: 13$ & -18.0 & $08: 29: 50$ & -17.9 \\
17 Aug 2001 & $16: 10: 10$ & -18.3 & $16: 49: 51$ & -18.2 \\
19 Aug 2001 & $18: 40: 05$ & -18.0 & $20: 09: 58$ & -18.3 \\
22 Aug 2001 & $08: 30: 08$ & -18.7 & $10: 29: 51$ & -18.5 \\
27 Aug 2001 & $01: 30: 07$ & -19.0 & $04: 29: 51$ & -18.8 \\
31 Aug 2001 & $16: 30: 15$ & -18.8 & $17: 29: 47$ & -19.0 \\
7 Sep 2001 & $20: 30: 10$ & -19.0 & $22: 58: 47$ & -19.1 \\
10 Sep 2001 & $07: 00: 12$ & -19.0 & $09: 29: 52$ & -19.0 \\
12 Sep 2001 & $13: 00: 11$ & -18.6 & $14: 39: 49$ & -18.9 \\
14 Sep 2001 & $22: 30: 48$ & -18.6 & $23: 59: 19$ & -18.8 \\
15 Sep 2001 & $00: 00: 16$ & -18.8 & $00: 59: 58$ & -18.8 \\
19 Sep 2001 & $12: 30: 09$ & -17.2 & $16: 29: 55$ & -18.3 \\
22 Sep 2001 & $04: 30: 11$ & -18.3 & $05: 29: 51$ & -18.2 \\
24 Sep 2001 & $07: 40: 14$ & -17.3 & $10: 19: 49$ & -17.8 \\
26 Sep 2001 & $22: 20: 16$ & -17.8 & $23: 14: 51$ & -17.7 \\
3 Oct 2001 & $23: 30: 14$ & -16.8 & $23: 59: 47$ & -16.8 \\
4 Oct 2001 & $00: 00: 07$ & -16.8 & $00: 59: 54$ & -16.8 \\
20 Oct 2001 & $09: 26: 46$ & -13.0 & $10: 16: 39$ & -13.4 \\
\hline
\end{tabular}

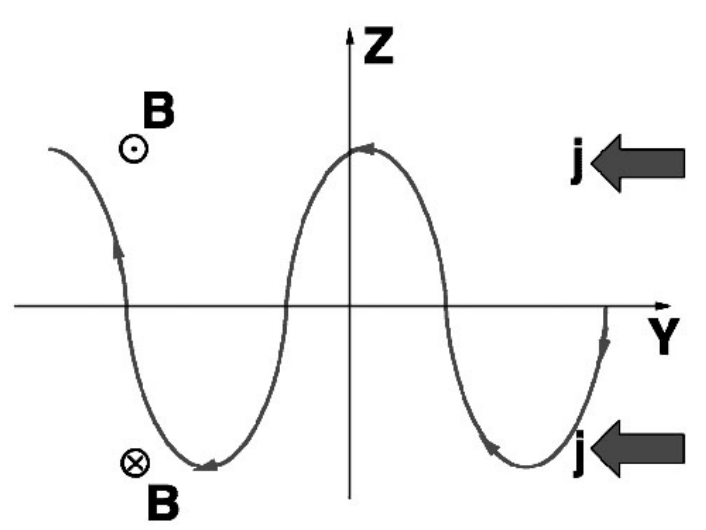

Fig. 1. Ion trajectory in the vicinity of the neutral layer. Associated electric current has two maxima at the distances $z$ of the order of ion gyroradius and is zero at $z=0\left(B_{x}=0\right)$.

et al., 2004). In most of these models, the current layer bifurcation is produced by non-adiabatic ion motion along the Speiser trajectories (Speiser, 1965). This effect is illustrated in Fig. 1. Magnetic field depends only on the z-coordinate and possesses only $B_{x}$-component, positive for $z>0$, and negative for $z<0$. Simplest type of Speiser ion orbit is shown for this configuration. This orbit arises when the ion velocity is directed along the $\mathrm{z}$-axis $\left(v_{y}=0\right)$ at $z=0$. At the distances of the order of gyroradius from $z=0$, the $v_{z}$-component vanishes, and ion velocity has only y-component, which has the same direction both for positive and negative $z$. This, in fact, is the splitting of the current sheet with the distance of the order of ion gyroradius between two peaks. It is possible to construct a self-consistent magnetic field distribution with $c / 4 \pi \operatorname{curl} \boldsymbol{B} \equiv \boldsymbol{j}=n e \boldsymbol{v}$, which reasonably well fits Cluster data even for this oversimplified model (Israelevich et al., 2007). The essential feature of this model, required for the current splitting, is the absence of $v_{y}$ at $z=0$, i.e. strong temperature anisotropy $\left(T_{y y} \neq T_{z z}\right)$.

In magnetized plasma, the temperature tensor (as well as the pressure tensor) in the coordinate system determined by the magnetic field direction takes diagonal form with, generally, three different temperatures, corresponding to three eigenvectors of the temperature tensor. Parallel temperature $T_{||}$is the tensor eigenvalue corresponding to the eigenvector parallel to the magnetic field, and two perpendicular temperatures $T_{\perp \text { max }}$ and $T_{\perp \text { min }}$ correspond to two other eigenvectors which are perpendicular to the magnetic field. In general case, $T_{\perp \text { max }} \neq T_{\perp \text { min }}$, and this type of temperature (and pressure) anisotropy is referred as non-gyrotropicity. The effect of the pressure non-gyrotropicity was incorporated in new kinetic studies of the current sheet structure (e.g. Sitnov et al., 2003). 
In this paper, we consider the dependence of the current density distribution on the ion temperature anisotropy.

\section{Method}

We use the results of electric current calculations as a curl of the magnetic field measured by FGM instruments aboard four Cluster (Balogh et al., 2001) satellites along with the calculations of the ion temperature from the distribution functions measured by CIS-CODIF instruments (Reme et al., 2001) aboard Cluster satellites 1, 3, and 4. We use current sheet crossings in August-October 2001 (see the list in Table 1). The 1-s averaged electric current density was calculated for the baricenter of satellites system. The $\mathrm{z}$-coordinate of neutral sheet (i.e. the point where $B_{x}=0$ ) varies permanently because of tail flapping. Let us use the coordinate system $X, Y, Z$ in which $X Y$ is the plane of the current sheet, the $Y$-axis is antiparallel to the current. Generally, magnetic field in the tail is a certain function of position, the set of solar wind parameters $S_{i}$ (like density, velocity, etc.), and, may be, the time:

$\boldsymbol{B}=\boldsymbol{B}\left(x(t), y(t), z(t), S_{i}(t) ; t\right)$

However, it is obvious that in the vicinity of the current sheet (i.e. close to $B_{x}=0$ ) the dependence of $B_{z}$ is dominant (as compared with dependencies on other parameters). The dependence $\boldsymbol{B}$ on changing solar wind parameters may be also neglected if the time period of the current sheet crossing is short. Therefore,

$$
B_{x}=B_{x}\left(x(t), y(t), z(t), S_{i}(t) ; t\right) \approx B_{x}(z)
$$

may be considered as a measure of the satellite (or baricenter) distance from the point of $B_{x}$-component reversal $(z=0)$ (of course, if $B_{x}$ increases monotonically with $z$ ). We analyze the electric current density distribution as a function of $B_{x}$. It is calculated by averaging measurements of the electric current density over $1 \mathrm{nT}$ bins of $B_{x}$. We consider only points where $\left|B_{x}\right|<25 \mathrm{nT}$ in order to eliminate strong currents which occasionally exist in the tail lobes. Electric current $\boldsymbol{j}$ is sorted by values of ion temperature measured at the same time as $\boldsymbol{j}$.

The ion temperature is calculated as follows. First, we calculate the ion number density as

$n=\int f(\boldsymbol{v}) d^{3} v$

( $f(\boldsymbol{v})$ is the distribution function), bulk velocity as

$V_{i}=\frac{1}{n} \int v_{i} f(\boldsymbol{v}) d^{3} v$

and pressure tensor as

$P_{i j}=m \int\left(v_{i}-V_{i}\right)\left(v_{j}-V_{j}\right) f(\boldsymbol{v}) d^{3} v$

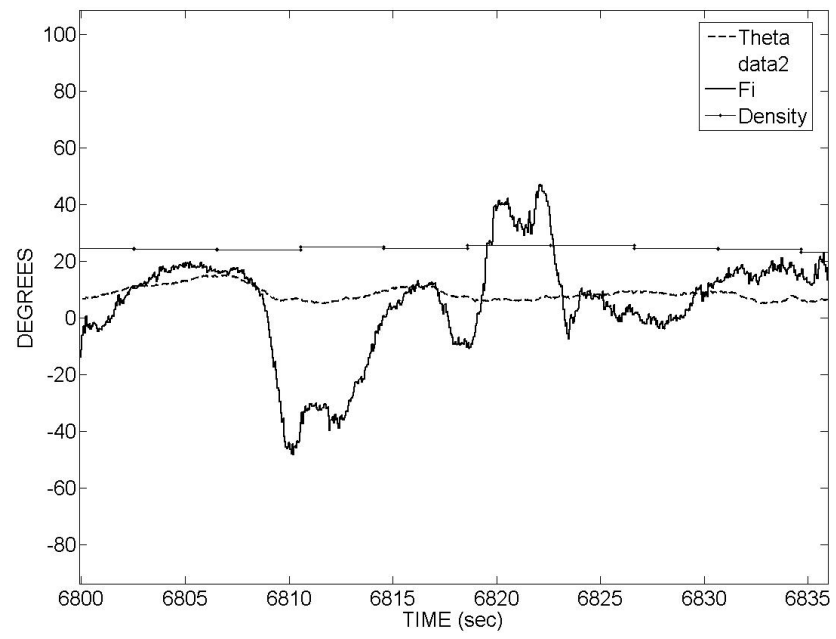

Fig. 2. Measurements of the ion number density (thin solid line with dots) along with the measurements of the polar angles $\varphi, \theta$ of the magnetic field vector. The direction of the magnetic field varies significantly during the time required to obtain a distribution function.

Then, the symmetric temperature tensor is

$T_{i j}=\frac{P_{i j}}{n}$

It is impossible to determine both the parallel and perpendicular temperatures directly, because, as it is shown in Fig. 2, the magnetic field may significantly change its direction during the time interval required for the distribution function measurement. Therefore we used the following procedure. First, at each moment, we calculate eigenvectors and eigenvalues of the temperature tensor thereby diagonalizing it. This way, we obtain set of three temperatures $T_{3}>T_{2}>T_{1}$. For ideal case of instantaneous measurements, one of the eigenvectors should be directed along the magnetic field vector, and the corresponding eigenvalue of temperature tensor is the parallel temperature $T_{\|}$, two other eigenvalues are $T_{\perp \text { min }}$ and $T_{\perp \text { max }}$. In real case, no one of the eigenvectors might be exactly parallel to the magnetic field. We have calculated the angle $\varphi$ as minimal of angles between three eigenvectors and the magnetic field vector. Figure 3 shows the probability density for the occurence of $\varphi$. The probability is normalized by $\sin \varphi$. Indeed, it has maximum near zero, therefore the eigenvalue corresponding to the eigenvector with minimal inclination to the magnetic field may be considered as a proxy for the parallel temperatures. This way we have sorted temperatures $T_{1}, T_{2}, T_{3}$ as $T_{\|}, T_{\perp \text { min }}$ and $T_{\perp \max }$.

\section{Results}

Figure 4 shows the results of averaging of all electric current density measurements over $1 \mathrm{nT}$ bins of $B_{x}$. There are two 


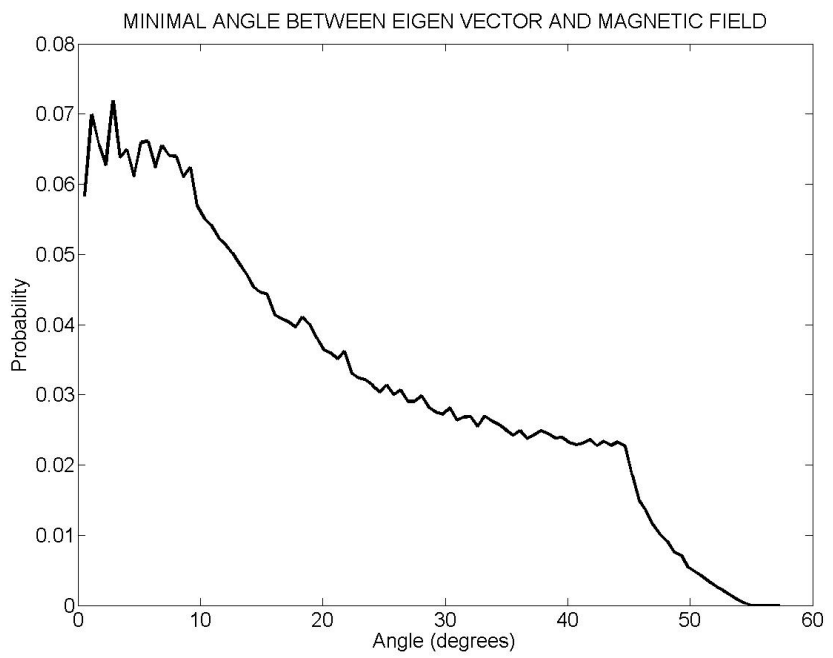

Fig. 3. Probability density for occurrence of the minimal angle $\varphi$ between the temperature tensor eigenvector and the magnetic field vector normalized by $\sin \varphi$.

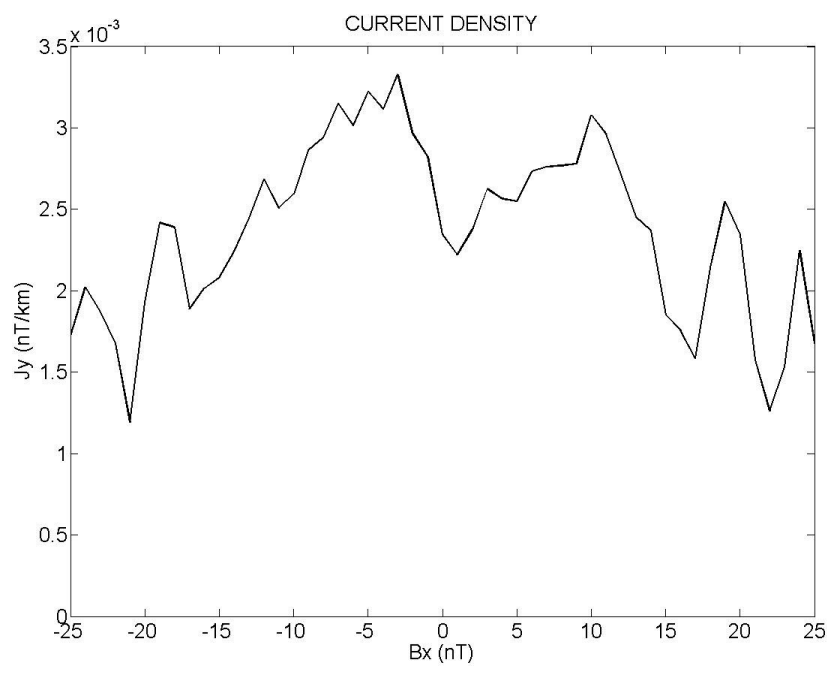

Fig. 4. The averaged (over the whole data) set distribution of the electric current versus the magnetic field component $B_{x}$.

double off-centered maxima of $j_{y}$. Thus, the averaged distribution of the electric current depicts the bifurcated current sheet as well as individual distributions of electric current density for some of geotail current sheet crossings. According to Asano et al. (2005), the majority of current sheet crossings is indicative of single-peak current density distribution, but distinctly double-peak current sheet crossings constitute a significant part (at least 17\%) of total number of crossings. The fact that double-peaked current sheet structure remains clearly visible after the averaging means that electric current density in double peaked current sheet is much stronger than in single-peaked one. If for given $B_{x}$-values, $j_{y}\left(B_{x}\right) \propto d B_{x} / d z$ is larger for one current sheet than for another, this means that

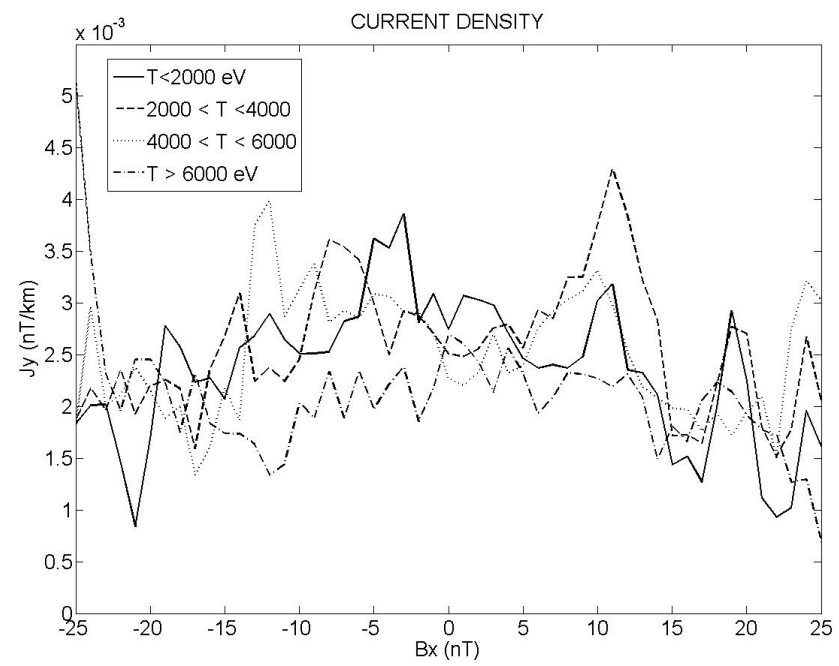

Fig. 5. The electric current density distributions $j_{y}\left(B_{x}\right)$ for different ion temperatures.

the first current sheet is thinner than the second. Thus bifurcated double-peaked sheets are expected to be thinner than Harris-type single-peaked sheets. Further analysis confirms this assumption.

First, we analyze the dependence of the current density profile on the temperature value. All the data set was sorted in four subsets, according to the measured temperature $T=\left(T_{1}+T_{2}+T_{3}\right) / 3$, namely, (1) data with $T<2 \mathrm{keV}$, (2) $2 \mathrm{keV}<T<4 \mathrm{keV}$, (3) $4 \mathrm{keV}<T<6 \mathrm{keV}$, and (4) $T>6 \mathrm{keV}$. For each subset, the averaging of the measured current density over $1 \mathrm{nT}$ bins of $B_{x}$ was made, and the dependencies $j_{y}\left(B_{x}\right)$ are shown in Fig. 5. All these distributions show the tendency to double-peak current sheet structure, but they are very similar and do not differ significantly from the average current density profile in Fig. 4. Thus we arrive at the conclusion that current sheet bifurcation does not depend on the ion temperature value.

Next, we consider the current profile structure for different anisotropies of the temperature. Let us introduce the temperature anisotropy factor as $R_{1}=2\left(T_{3}-T_{1}\right) /\left(T_{3}+T_{1}\right)$. If we sort the data set in four subsets, according to the value of $R_{1}$ : (1) $R_{1}<0.2$; (2) $0.2<R_{1}<0.4$; (3) $0.4<R_{1}<0.6$ and (4) $R_{1}>0.6$, the difference between the current density profiles becomes distinct - see Fig. 6. For the subset of measurement points with isotropic temperature $\left(R_{1}<0.2\right)$, the electric current density has a single-peak profile, with maximal current density of $3 \times 10^{-3} \mathrm{nT} / \mathrm{km}\left(2.4 \mathrm{nA} / \mathrm{m}^{2}\right)$. All subsets with strong temperature anisotropy $\left(R_{1}>0.2\right)$ correspond to double-peaked bifurcated current sheet. Moreover, the maximal electric current density systematically increases with the growth of the anisotropy, reaching $8 \times 10^{-3} \mathrm{nT} / \mathrm{km}$ $\left(6.4 \mathrm{nA} / \mathrm{m}^{2}\right)$ for $R_{1}>0.6$.

On the contrary, current sheet structure does not depend on the fact whether or not the intermediate eigenvalue of 


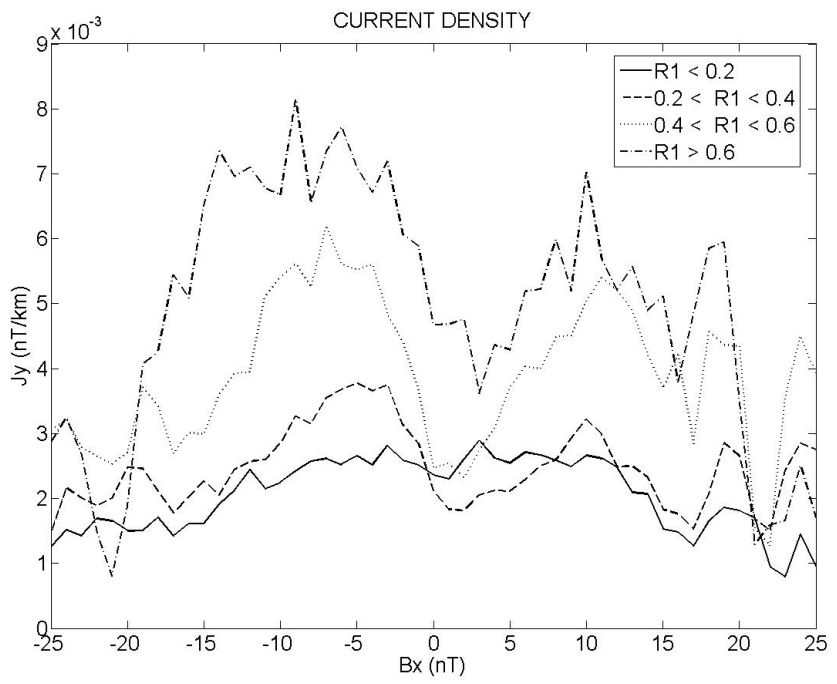

Fig. 6. The electric current density distributions $j_{y}\left(B_{x}\right)$ for different temperature anisotropy ratios $R_{1}$.

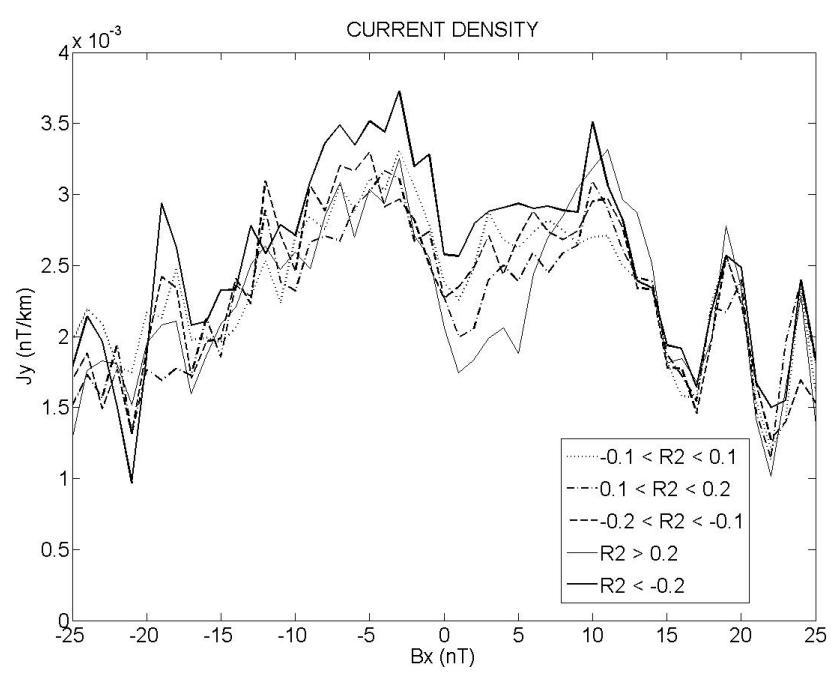

Fig. 7. The electric current density distributions $j_{y}\left(B_{x}\right)$ for different temperature anisotropy ratios $R_{2}$.

temperature $T_{2}$ is close to maximal eigenvalue $T_{3}$ or to minimal eigenvalue $T_{1}$, as it is characterized by the anisotropy ratio $R_{2}=\frac{T_{2}-\left(T_{1}+T_{3}\right) / 2}{\left(T_{1}+T_{2}+T_{3}\right) / 3}$. Figure 7 shows the function $j_{y}\left(B_{x}\right)$ for different ratios $R_{2}$ : (1) $R_{2}<-0.2$; (2) $-0.2<R_{2}<-0.1$; (3) $-0.1<R_{2}<0.1$; (4) $0.1<R_{2}<0.2$ and (5) $R_{2}>0.2$. All current profiles are very similar.

Therefore, bifurcation of geotail current sheet is observed when the ion temperature is anisotropic. As mentioned above, there are two different types of temperature anisotropy - the anisotropy of parallel and perpendicular to the magnetic field temperatures characterized by the ratio $T_{\|} / T_{\perp}$, and the anisotropy of perpendicular temperature (non-gyrotropicity) $T_{\perp \max } / T_{\perp \text { min }}$. In order to check, which

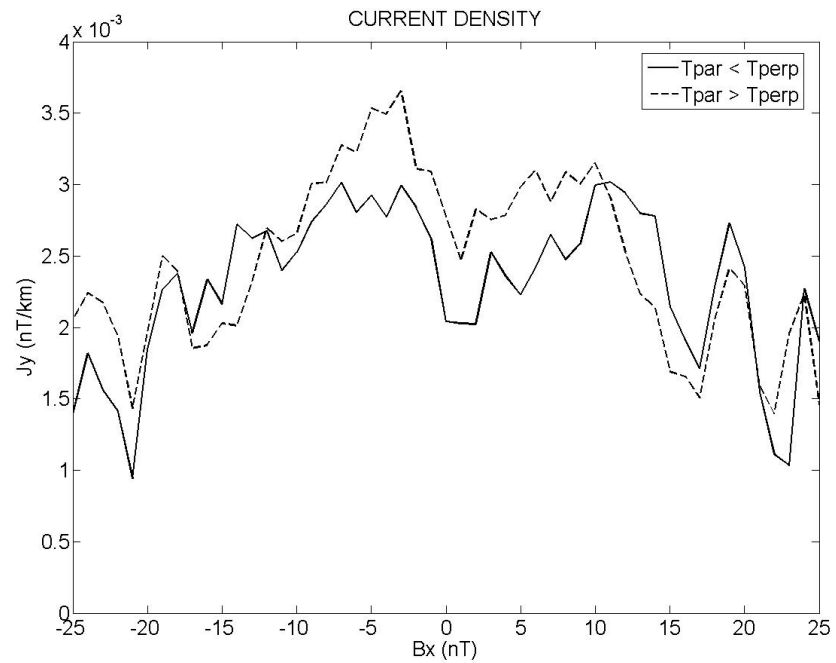

Fig. 8. The electric current density distributions $j_{y}\left(B_{x}\right)$ for $T_{\|}<T_{\perp}$ (solid line) and $T_{\|}>T_{\perp}$ (dashed line).

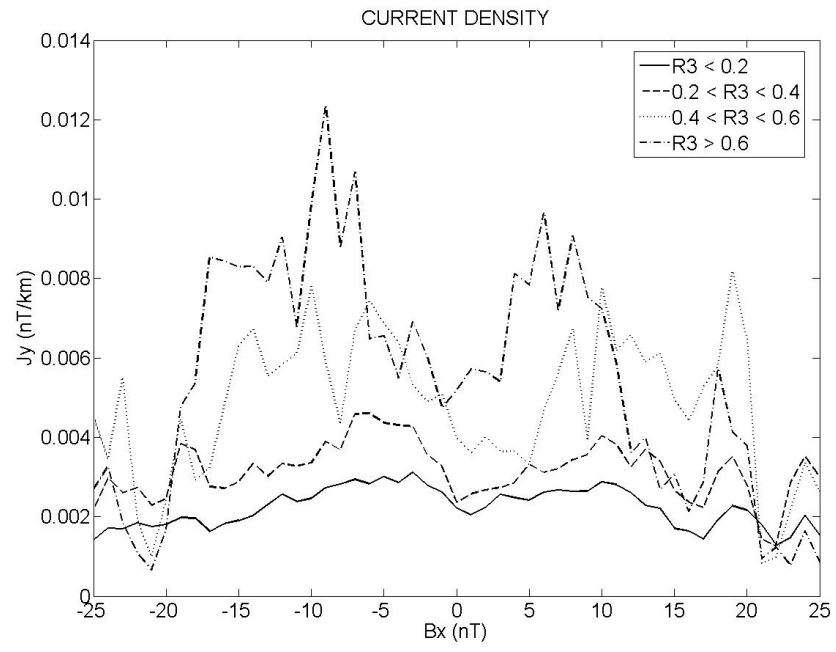

Fig. 9. The electric current density distributions $j_{y}\left(B_{x}\right)$ for different temperature anisotropy ratios $R_{3}$.

of them is responsible for the current sheet bifurcation we will use the sorted data set of temperature eigenvalues (described above), where the parallel temperature $T_{\|}$is determined as the eigenvalue corresponding to the eigenvector with smallest inclination to the magnetic field direction.

Figure 8 show current sheet profiles for $T_{\|}<T_{\perp}$ and $T_{\| \mid}>T_{\perp}$. According to Cowley (1978) double-peaked current sheet exists in the first case, and single-peak current sheet in the second one. It is not true for the considered Cluster data. Both profiles in Fig. 8 are double-peaked, and, moreover, practically identical.

On the contrary, if we introduce non-gyrotropicity factor $R_{3}=\frac{T_{\perp \max }-T_{\perp \min }}{\left(T_{\perp \max }+T_{\perp \min }\right) / 2}$, the data subsets sorted by $R_{3}$ values exhibit strong change of the current sheet structure 

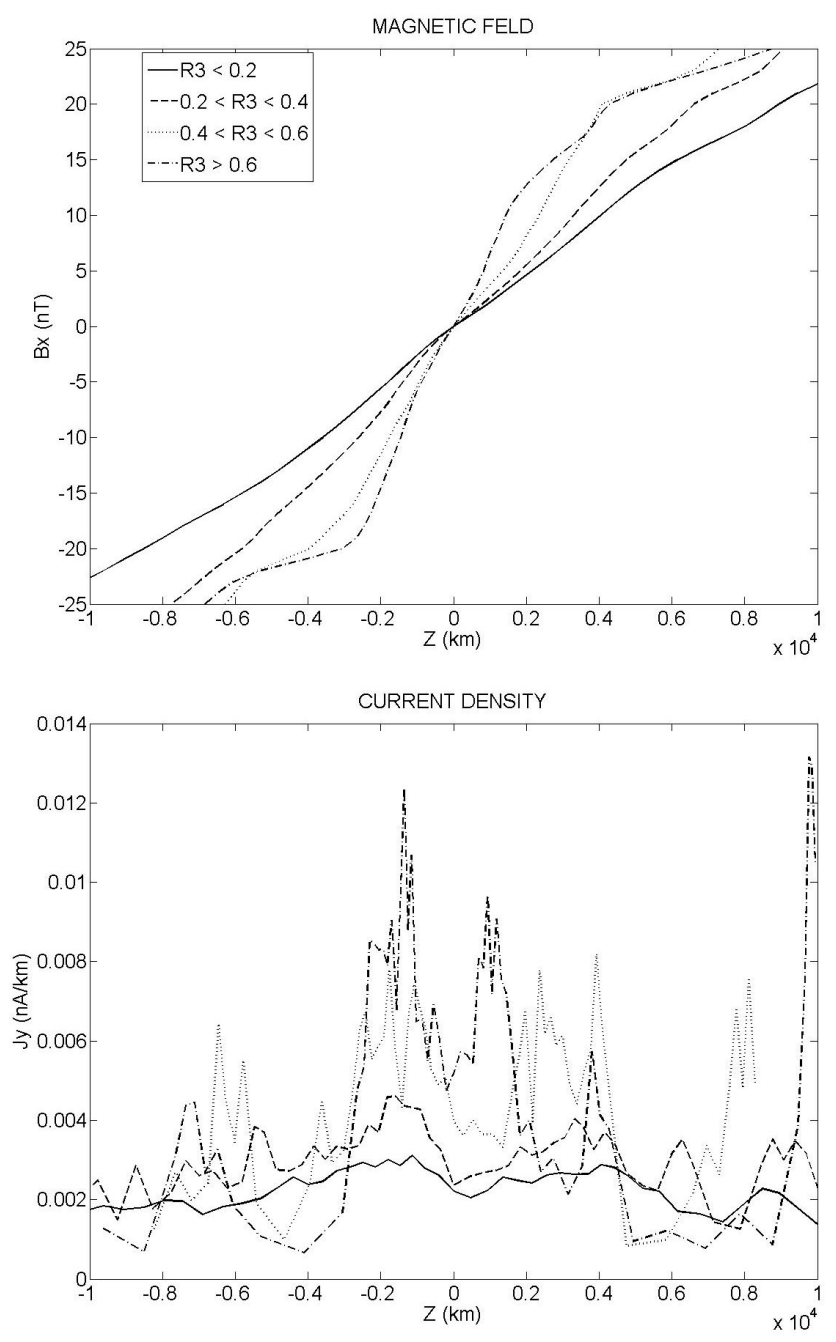

Fig. 10. Profiles of the magnetic field $B_{x}(z)$ (top) and the electric current density distributions $j_{y}(z)$ in the geotail current sheet for different temperature anisotropy ratios $R_{3}$.

versus $R_{3}$, as shown in Fig. 9. The profiles are given for $R_{3}<0.2 ; 0.2<R_{3}<0.4 ; 0.4<R_{3}<0.6$ and $R_{3}>0.6$. Comparison with Fig. 6 shows that sorting of the temperature eigenvalues via the magnetic field direction enhances sensitivity of the current structure to the anisotropy value. In Fig. 6, where effects of parallel-to-perpendicular and perpendicular anisotropy (non-gyrotropicity) are mixed together, the average current density distribution is single-peaked for $R_{1}<0.2$, whereas for $R_{3}<0.2$ (Fig. 9) one may still distinguish double-peaked structure. For large anisotropies, unsorted set gives maximum current density $8 \times 10^{-3} \mathrm{nT} / \mathrm{km}$ $\left(6.4 \mathrm{nA} / \mathrm{m}^{2}\right)$ for $R_{1}>0.6$, and for sorted data set maximal current is $12 \times 10^{-3} \mathrm{nT} / \mathrm{km}\left(9.6 \mathrm{nA} / \mathrm{m}^{2}\right)$. Thus, our method of sorting, indeed, refines the data set.

Magnetic field and current density profile in real geometric coordinates may be restored from the current density distri- butions shown in Fig. 9, by integration

$z=\int \frac{c d B_{x}}{4 \pi j_{y}\left(B_{x}\right)}+$ Const

The results, shown in Fig. 10, confirm the above assumption about relationship between current sheet thickness and bifurcation. Current sheet thickness decreases when non-gyrotropicity factor $R_{3}$ grows, reaching $\sim 4000 \mathrm{~km}$ for $R_{3}>0.6$. One should keep in mind, that the averaged electric current density is smaller (almost by a factor 5) than electric current density for some individual crossings. The current sheet thickness for such extremely strong current density may be as small as $1000 \mathrm{~km}$.

\section{Conclusions}

Current density distribution in the geomagnetic tail is shown not to depend on the ratio between the parallel and perpendicular ion temperature. Double-peaked as well as singlepeaked distributions are observed both for the case $T_{||}>T_{\perp}$ and for $T_{\|}<T_{\perp}$. The average distributions of current density are shown to be the same for both cases.

We have shown that tail current sheet bifurcation is controlled by non-gyrotropicity of plasma pressure: double peaked current density distribution is observed when the ion perpendicular temperature exhibits anisotropy, and the electric current density is stronger for larger ratio $\cdot\left(T_{\perp \max }-T_{\perp \min }\right) / T_{\perp}$. Generally, double-peaked current sheets are thinner than single-peaked sheets. The current sheet thinning is accompanied by the perpendicular temperature anisotropy.

Acknowledgements. Authors are grateful to A. Runov for stimulating discussions. We also appreciate Cluster Active Archive at http://caa.estec.esa.int, and Cluster project FGM and CIS teams.

Topical Editor I. A. Daglis thanks two anonymous referees for their help in evaluating this paper.

\section{References}

Asano, Y., Nakamura, R., Baumjohann, W., Runov, A., Vörös, Z., Volwerk, M., Zhang, T. L., Balogh, A., Klecker, B., and Rème, H.: How typical are atypical current sheets?, Geophys. Res. Lett, 32, L03108, doi:10.1029/2004GL021834, 2005.

Balogh, A., Carr, C. M., Acuna, M.-H., Dunlop, M. W., Beek, T. J., Brown, P., Fornacon, K.-H., Georgescu, E., Glassmeier, K.-H., Harris, J. P., Musmann, G., Oddy, T. M., and Schwingenschuh, K.: The Cluster magnetic field investigation: Overview of inflight performance and initial results, Ann. Geophys., 19, 12071217, 2001, http://www.ann-geophys.net/19/1207/2001/.

Behannon, K. W.: Geometry of the geomagnetic tail, J. Geophys. Res., 75, 743-753, 1970.

Camporeale, E. and Lapenta, G.: Model of bifurcated current sheets in the Earth's magnetotail: equilibrium and stability, J. Geophys. Res., 110, A07206, doi:10.1029/2004JA010779, 2005. 
Cowley, S. W. H.: The effect of pressure anisotropy on the equilibrium structure of magnetic current sheets, Planet. Space Sci., 26, 1037-1061, 1978.

Daughton, W., Lapenta, G., and Ricci, P.: Nonlinear evolution of the lower-hybrid drift instability in a current sheet, Phys. Rev. Letts., 93(10), 105 004, doi:10.1103/PhysRevLett.93.105004, 2004.

Fairfield, D. H.: On the average configuration of the geomagnetic tail, J. Geophys. Res., 84, 1950-1958, 1979.

Genot, V., Mottez, F., Fruit, G., Louarn, P., Sauvaud, J. A., and Balogh, A.: Bifurcated current sheet: model and Cluster observations, Planet. Space Sci., 53, 229-235, 2005.

Harris, E. G.: On a plasma sheet separating regions of oppositely directed magnetic field, Nuovo Cimento, 23, 115-121, 1962.

Hoshino, M., Nishida, A., Mukai, T., Saito, Y., Yamamoto, T., and Kokubun, S.: Structure of plasma sheet in magnetotail: Doublepeaked electric current sheet, Geophys. Res., 101, $24775-$ $24786,1996$.

Israelevich, P. and Ershkovich, A.: Bifurcation of Jovian magnetotail current sheet, Ann. Geophys., 24, 1479-1481, 2006, http://www.ann-geophys.net/24/1479/2006/.

Israelevich, P. L., Ershkovich, A. I., and Oran, R.: Bifurcation of the Tail Current Sheet in Jovian Magnetosphere, Planet. Space Sci., 55, 2261-2266, 2007.

Israelevich, P. L., Ershkovich, A. I., and Oran, R.: Current carriers in the bifurcated current sheet: electrons or ions?, J. Geophys. Res., 113, A04215, doi:10.1029/2007JA012541, 2008.

Karimabadi, H., Daughton, W., Pritchett, P. L., and Krauss-Varban, D.: Ion-ion kink instability in the magnetotail: 1. Linear theory, J. Geophys. Res., 108, 1400, doi:10.1029/2003JA010026, $2003 \mathrm{a}$.

Karimabadi, H., Pritchett, P. L., Daughton, W., and KraussVarban, D.: Ion-ion kink instability in the magnetotail: 2. Three-dimensional full particle and hybrid simulations and comparison with observations, J. Geophys. Res., 108, 1401, doi:10.1029/2003JA010109, 2003b.

Reme, H., Aoustin, C., Bosqued, J. M., et al.: First multi-spacecraft ion measurements in and near the Earth's magnetosphere with the identical Cluster ion spectrometry (CIS) experiment, Ann. Geophys., 19, 1303-1354, 2001, http://www.ann-geophys.net/19/1303/2001/.
Ricci, P., Lapenta, G., and Brackbill, J. U.: Structure of the magnetotail current: Kinetic simulation and comparison with satellite observations, Geophys. Res. Lett., 31, L06801, doi:10.1029/2003GL019207, 2004.

Runov, A., Nakamura, R., Baumjohann, W., Zhang, T. L., Volwerk, M., Eichelberger, H.-U., and Balogh, A.: Cluster observation of a bifurcated current sheet, Geophys. Res. Lett., 30, 1036, doi:10.1029/2002GL016136, 2003.

Runov A., Sergeev, V., Nakamura, R., Baumjohann, W., Vörös, Z., Volwerk, M., Asano, Y., Klecker, B., Rème, H., and Balogh, A.: Propeties of a bifurcated current sheet observed on 29 August 2001, Ann. Geophys., 22, 2535-2540, 2004, http://www.ann-geophys.net/22/2535/2004/.

Runov, A., Sergeev, V., Nakamura, R., Baumjohann, W., Zhang, T. L., Asano, Y., Volwerk, M., Vörös, Z., Balogh, A., and Rème, H.: Reconstruction of the magnetotail current sheet structure using multi-point Cluster measurements, Planet. Space Sci., 53, 237243, 2005.

Runov, A., Sergeev, V., Nakamura, R., Baumjohann, W., Apatenkov, S., Asano, Y., Takada, T., Volwerk, M., Vörös, Z., Zhang, T. L., Sauvaud, J. A., Rème, H., and Balogh, A.: Local structure of the magnetotail current sheet: 2001 Cluster observations, Ann. Geophys., 24, 247-262, 2006, http://www.ann-geophys.net/24/247/2006/.

Sergeev, V. A., Mitchell, D. G., Russell, C. T., and Williams, D. J.: Structure of the tail plasma/current sheet at $\sim 11 R_{E}$ and its changes in the course of a substorm, J. Geophys. Res., 98, 17 345-17 365, 1993.

Sergeev, V., Runov, A., Baumjohann, W., et al.: Current sheet flapping motion and structure observed by Cluster, Geophys. Res. Lett., 30, 1327, doi:10.1029/2002GL016500, 2003.

Sitnov, M. I., Guzdar, P. N., and Swisdak, M.: A model of the bifurcated current sheet, Geophys. Res. Lett., 30, 1712, doi:10.1029/2003GL017218, 2003.

Speiser, T. W.: Particle Trajectories in Model Current Sheets, 1, Analytical Solutions, J. Geophys. Res., 70, 4219-4226, 1965.

Zelenyi, L. M., Malova, H. V., and Popov, V. Yu.: Splitting of thin current sheets in the Earth's magnetosphere, JETP Lett., 78, 296299, 2003. 